\title{
On Teaching Switched Mode Power Supplies - A Converter with Limited Duty Cycle
}

\author{
Felix A. Himmelstoss ${ }^{\dagger}$ \\ $\dagger$ Dept. of Energy and Industrial Electronics, University of Applied Science Technikum Wien, Vienna, Austria
}

\begin{abstract}
A way how to teach a general understanding of switched mode power supplies (SMPS) is shown. A fourth order PWM DC-to-DC converter with limited duty cycle range is treated as an example and a survey over important data (maximum voltage and current ratings for the elements, rms- values for the semiconductor devices and a rough approximation of the losses) of the circuit is given. Furthermore, a converter model based on duty ratio averaging is established. Continuous mode of operation is used. The results make it possible to estimate the applicability of the given converter structure and offer sufficient material for the calculation, design, and analysis and give a better insight into switched mode energy conversion.
\end{abstract}

Key Words: DC-to-DC converter, Modeling, Switched mode power supply

\section{INTRODUCTION}

Teaching switched mode power supplies is a sophisticated task. There exists a comprehensive treatment of this topic in the textbooks e.g. [1 till 6] and there are numerous papers in conference proceedings and journals. On the other hand you only need to know what an inductor and a capacitor really are, that means to understand the meaning of

$$
\begin{aligned}
& u_{L}=L \cdot \frac{d i_{L}}{d t}, \\
& u_{C}=\frac{1}{C} \int_{0}^{t} i_{C} \cdot d t+u_{C}(0) \\
& u_{R}=R \cdot i_{R} .
\end{aligned}
$$

Furthermore, Kirchhoff's current law (KCL) and Kirchhoff's voltage law (KVL) have to be applied correctly. To get a knowledge of the general function of a switched mode power supply, the semiconductor devices can be interpreted as simple switches. There is (at least) one active switch (bipolar junction transistor, MOSFET, IGBT), which can be arbitrarily turned on and off and at least one passive switch (diode), which turns on and off according to outer conditions. With this in mind it is possible to analyze the function of an SMPS with more than one inductor and capacitor.

The "learning by doing"- concept can be used to obtain a better understanding of how converters work. Starting from the classical converters (buck, boost, buck-boost, and the cuk as an example for fourth-order converters), the students learn by

\footnotetext{
Manuscript received Apr. 29, 2010; revised Oct. 15, 2010

$\dagger$ Corresponding Author: himmelstoss@technikum-wien at

Tel: +43-1-3334077-229, Fax: +43-1-3334077-236, Univ. of Applied Science Technikum Wien

Dept. of Energy and Industrial Electronics, University of Applied Science Technikum Wien, Austria
}

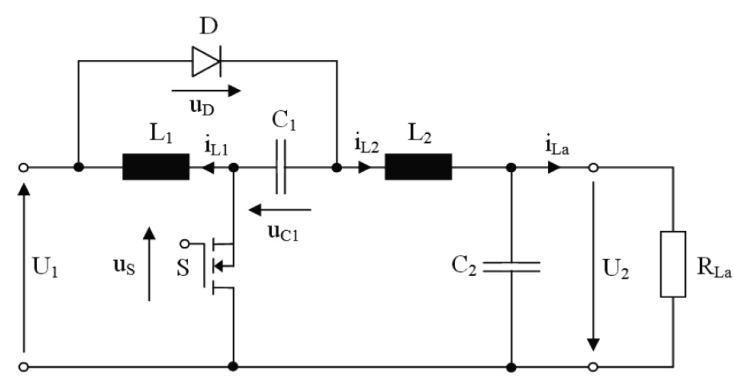

Fig. 1. DC-to-DC converter.

means of lectures about the converters and how to treat them. In a next step they have to analyze a family of 16 fourthorder converters with restricted duty cycle [7] on their own in a way similar to the one shown here. These converters are not so well-known and therefore the students must make the analysis on their own. Two students form a group which works together. In this paper an example (Fig.1) for such an analysis is shown.

The first problem to be solved is the choice of the current (through the inductors) and the voltage (across the capacitors) senses. The direction of the current through and the voltage across the devices are the same with the exception of uncontrolled sources. (For us the arrowhead for the voltage means minus, that is different in other countries; voltages are denominated with $\mathrm{u}$ (small letter) for time varying and with $\mathrm{U}$ (capital letter) for constant values.) The voltage at the input and output terminals is usually known. Under the assumption that the circuit is performing as a converter, the direction of the currents through the inductors must be in such a sense, that after turn-off of the active switch the currents through the inductors can commutate into the diode branch. The voltages 

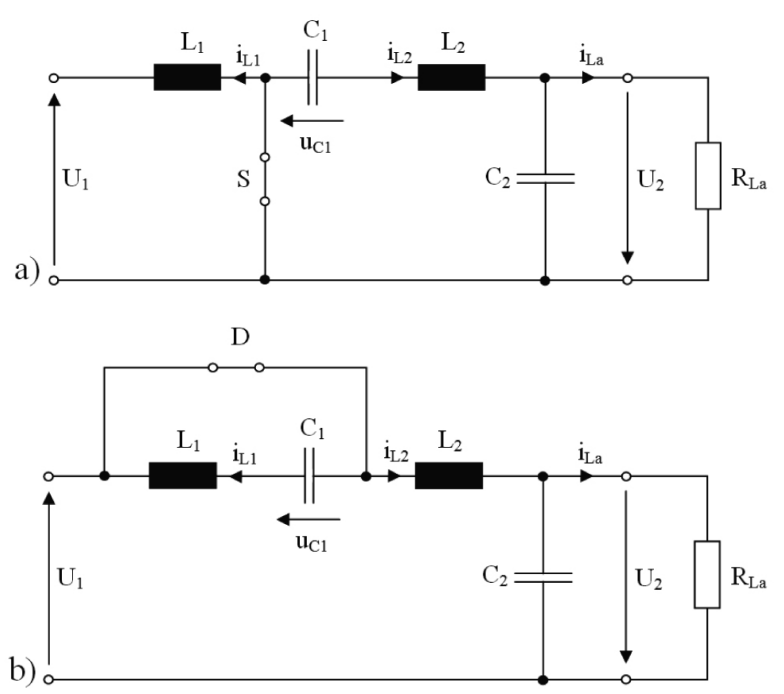

Fig. 2. Equivalent circuit of the DC/DC converter, a. turn-on switching state, b. turn-off switching state.

across the capacitors have to be chosen in such a way, that the diode is in blocking condition when the active switch is turned on. In steady-state there must be a volt-second balance, that means the positive and the negative voltage-time product across the inductors during one switching cycle are the same.

\section{BASIC ANALYSIS}

The basic analysis has to be done with idealized components (that means no parasitic resistors, no switching losses) and for the continuous mode in steady (stationary) state condition. A good way to start is to consider the voltage across the inductors.

Fig. 2 shows the equivalent circuit of the converter for conducting active switch (Fig.2(a)) and for conducting passive switch (Fig.2(b)). When the switch $\mathrm{S}$ is turned off the current through the inductors find there way by turning on the diode D.

Since for the stationary case the absolute values of the voltsecond balance of the inductors have to be equal (the voltage across the inductor has to be zero in the average), we can easily draw the shapes according to Fig.3. (Here, the capacitors are assumed so large that the voltages can be regarded constant during a pulse period.) Fig. 3(a) shows the voltage across and the current through L1. The current slope of course depends on the values of L1 and U1. Fig. 3(b) shows the current through and the voltage across L2. For L1 = L2, the same current ripple occurs in both inductors.

Based on the equality of the voltage-time-areas in the stationary case, it is easy to give the transformation relationship for the output voltage U2 dependent on the input voltage U1 and the duty ratio $\mathrm{d}$, which is the ratio between the on-time of the active switch and the switching period $\left(d=T_{O N} / T\right)$.

From Fig. 3.a. we get

$$
d \cdot U_{1}=(1-d) \cdot U_{c 1}
$$

and from Fig.3.b

$$
d \cdot\left(U_{c 1}-U_{c 2}\right)=\left(U_{1}+U_{C 2}\right) \cdot(1-d)
$$
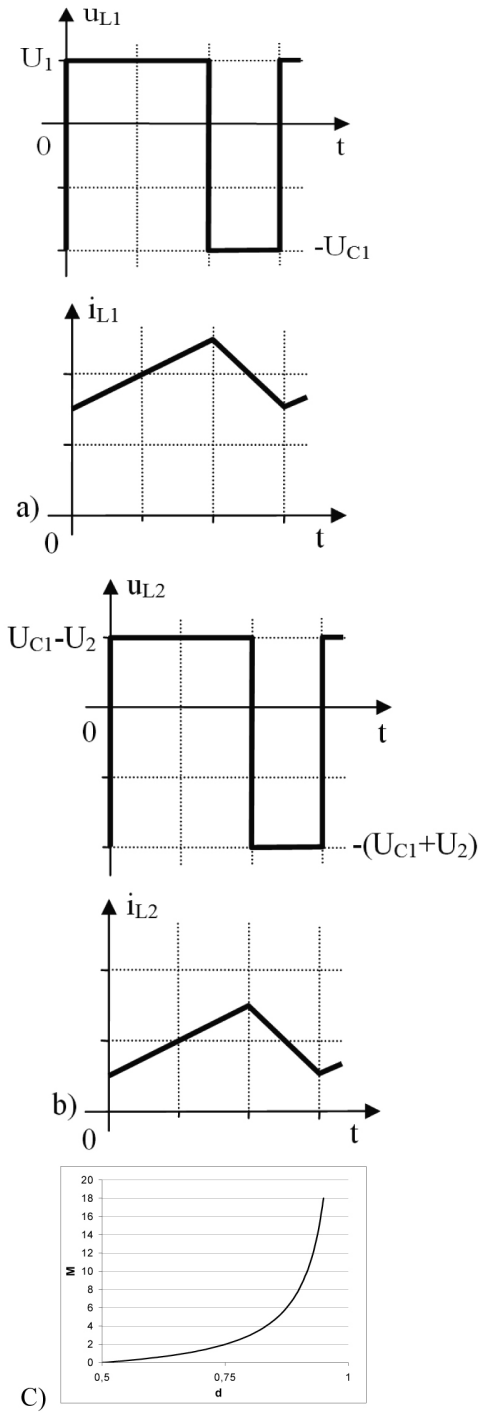

Fig. 3. Voltages across and current through the inductors L1 and L2, a. L1, b. L2; (c).Voltage transformation factor (static conversion ratio) $M$ in dependence on the duty cycle $d$.

After a few steps we get the voltage transformation law

$$
U_{C 2}=U_{2}=M \cdot U_{1}=\frac{1-2 \cdot d}{d-1} \cdot U_{1} \text { with } d \in[0.5,1[.
$$

Fig. 3(c) shows the static conversion ratio $M$ (the ratio of output voltage to input voltage) in dependence on the duty cycle d. The converter is a step - down/up converter with restricted duty cycle due to the fact, that a duty cycle lower than 0.5 would lead to an inversion of the output voltage. One has to have in mind, however, that due to the losses of the real converter the step-up rate is limited (to about 3 ).

In the same manner a connection for the current through the inductors can be derived based on the equality of the absolute values of the current-time-areas of the capacitors during onand off-times of the active switch.

From Fig.4(a) we get for the average values of the inductor currents $\bar{I}_{L 1}$ and $\bar{I}_{L 2}$

$$
(1-d) \cdot \bar{I}_{L 1}=d \cdot \bar{I}_{L 2} .
$$



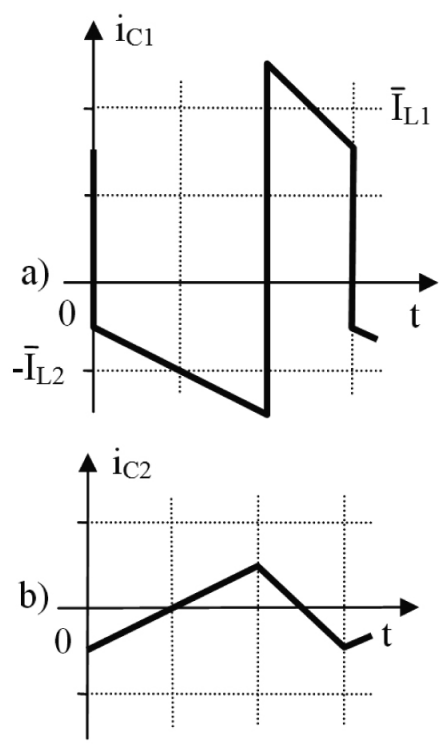

Fig. 4. Current through the capacitors $\mathrm{C} 1$ (a) and $\mathrm{C} 2$ (b).

Have in mind that the current ripple should be not too high in practice to avoid too high current peak values. On the other side, according to Fig.1 we can write

$$
\bar{I}_{L 2}=I_{\text {Load }} \text {. }
$$

(the current through the capacitor has to be an AC current, otherwise the output voltage would change). The current through according to (5) and (6) is

$$
\bar{I}_{L 1}=\frac{d}{1-d} \cdot I_{\text {Load }} \text {. }
$$

Furthermore, it is easy to construct the voltages across and the currents through the active and the passive switch (Fig.5) and therefore one can calculate the maximum ratings for the semiconductor devices. It is also important to calculate the relationship between the input current, the load current and the currents through the inductors to get dimensioning information.

From the equivalent circuits one can immediately see, that the current through the semiconductor devices is the sum of the inductor currents (through the active switch during Ton, through the passive switch during $\mathrm{T}_{\text {off }}$ ). Therefore, the current maximum values (neglecting the current ripple, middle height of the trapezoid in Fig.5 and Fig.6) for the semiconductor devices are

$$
\hat{I}_{D S, \text { max }}=\hat{I}_{A K, \text { max }}=\left(\bar{I}_{L 1}+\bar{I}_{L 2}\right)=I_{\text {Load }} \cdot \frac{1}{1-d} .
$$

For the calculation of the on-state losses the rms values are important. For the diode we get approximately (we avoid the integration of the square of the trapezoid-shaped currents. This is correct when large inductors are used, which lead to small current ripples)

$$
I_{A K, r m s}=I_{L o a d} \cdot \frac{1}{\sqrt{1-d}}
$$

and for the transistor

$$
I_{D S, r m s}=I_{\text {Load }} \cdot \frac{\sqrt{d}}{1-d} .
$$
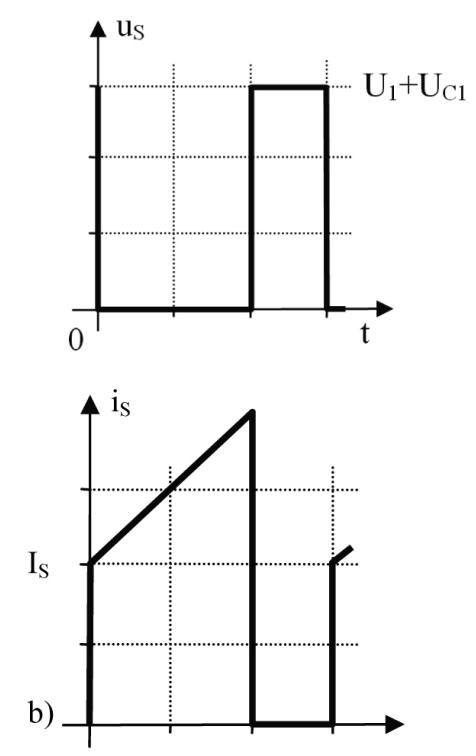

Fig. 5. Voltage across (a) and current through (b) the active switch.

Fig. 5 shows the voltage across and the current through the active switch (MOSFET) Fig. 6 shows the same for the diode. The input current shape can be easily calculated from $i_{1}=i_{D S}-i_{L 2}$ (Fig.7). One can see clearly, that the converter feeds back energy into the energy source during the on-time of the diode. There is also a disadvantage because of the switched nature of the input current, leading to an appropriate large capacitor parallel to the input voltage source (especially when batteries are used).

For the dimensioning of the circuit the voltage rates for the semiconductors are important. According to the equivalent circuit for the on-state ( $\mathrm{S}$ on, D off, Fig.2(a)), the voltage across the diode is

$$
U_{A K, \max }=-\left(U_{1}+U_{C 1}\right)=-U_{1} \cdot \frac{1}{1-d}=-\left(2 \cdot U_{1}+U_{2}\right)
$$

and according to Fig.2(b)(S off, D on) the voltage across the active switch is

$$
U_{D S, \max }=U_{1}+U_{C 1}=U_{1} \cdot \frac{1}{1-d}=2 \cdot U_{1}+U_{2}
$$

Therefore, the voltage ratings for the semiconductor devices must be larger than twice the input voltage plus the output voltage. Fig. 5 and Fig. 6 show this relation graphically.

For continuous operation mode and synchronous rectification it is easy to calculate a rough approximation of the efficiency of the converter. With $r_{D}$ as on-resistance of the semiconductors we can calculate the conduction losses (without switching losses) according to

$$
P_{V}=r_{D} \cdot\left(\frac{I_{\text {Load }}}{1-d}\right)^{2}
$$

and further get a rough estimate of the efficiency with the equation

$$
\eta=\frac{\left(U_{2} \cdot I_{\text {Load }}\right)}{\left(U_{2} \cdot I_{\text {Load }}\right)+P_{v}}=\frac{1}{1+r_{D} \cdot \frac{I_{L o a d} \cdot\left(U_{2}+2 \cdot U_{1}\right)^{2}}{U_{2} \cdot U_{1}^{2}}}
$$


a)

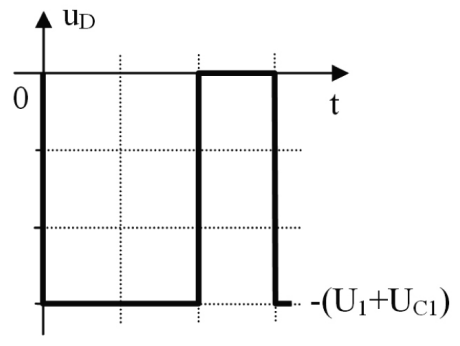

b)

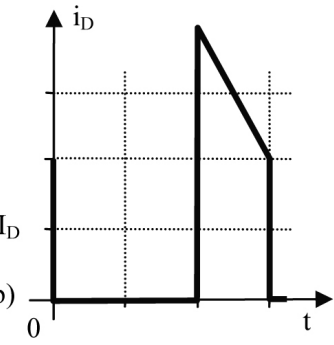

Fig. 6. Voltage across and current through the passive switch

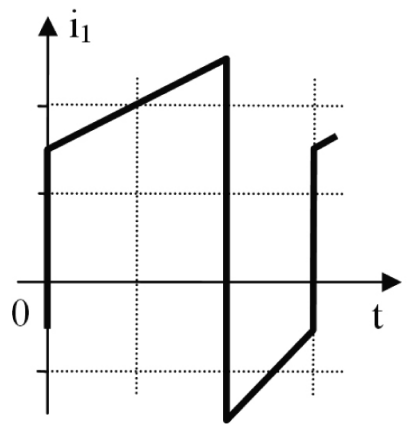

Fig. 7. Input current of the converter.

From these basic considerations one can already see the most essential aspects of the circuit. Because the duty ratio for the converter has to be always larger than 0.5 , it is obvious to use a snubber for reducing the switching losses in the active switches in these cases. The typical active snubber uses resonant circuits for discharging the snubber capacitor during the on-time of the switch. There is always at least half of the pulse period available for discharging the snubber capacitor(s) thus making it easily possible to dimension a low-loss snubber network. There is no restriction of the duty ratio necessary due to the snubber network as would the case of a converter with a duty cycle range of theoretically [0,1]. Thereby higher switching frequencies become realizable. A second interesting aspect of the circuit is given by the fact, that the voltage is equal across both inductors. This makes possible a coupling of L1 and L2 and therefore to have only one magnetic part in the circuit (integrated magnetics) [8].

\section{DyNAMIC MODEL REPRESENTATION}

For the sake of brevity the consideration of the following details shall be omitted: the influence of the ESR (equivalent series resistance) of the capacitors and of the ohmic contribution of the inductive storage element; also ideal switches are assumed. However, only a model with loss resistance gives good consistency with practical experiments and describes the dynamic correctly. It is given in the appendix.

One can establish two describing differential equation systems for closed active switch and for open switch respectively. These two systems can be combined into one system of differential equations. Then this system of nonlinear differential equations can be linearized about its operating point enabling us to use the linear control theory. During the interval Ton the equivalent circuit of Fig.2(a) is valid, leading to the equations

$$
\begin{aligned}
\frac{d i_{L 1}}{d t} & =\frac{u_{1}}{L_{1}} \\
\frac{d i_{L 2}}{d t} & =\frac{u_{C 1}-u_{C 2}}{L_{2}} \\
\frac{d u_{C 1}}{d t} & =\frac{-i_{L 2}}{C_{1}} \\
\frac{d u_{C 2}}{d t} & =\frac{i_{L 2}-u_{C 2} / R}{C_{2}}
\end{aligned}
$$

During the interval Toff the equivalent circuit of Fig.2(b) is applicable; the corresponding equations are

$$
\begin{aligned}
\frac{d i_{L 1}}{d t} & =\frac{-u_{C 1}}{L_{1}} \\
\frac{d i_{L 2}}{d t} & =\frac{-u_{1}-u_{C 2}}{L_{2}} \\
\frac{d u_{C 1}}{d t} & =\frac{i_{L 1}}{C_{1}} \\
\frac{d u_{C 2}}{d t} & =\frac{i_{L 2}-u_{C 2} / R}{C_{2}}
\end{aligned}
$$

(15) and (16) describe the system behavior. Under the condition, that the system time constants are large compared to the switching period, we can combine these two sets of equations. The duty ratio shall be defined as

$$
d=\frac{T_{o n}}{T}
$$

Weighed by this duty ratio, the combination of the two sets yields to the state-space model

$$
\frac{d}{d t}\left(\begin{array}{c}
i_{L 1} \\
i_{L 2} \\
u_{C 1} \\
u_{C 2}
\end{array}\right)=\left[\begin{array}{cccc}
0 & 0 & \frac{d-1}{L_{1}} & 0 \\
0 & 0 & \frac{d}{L_{2}} & -\frac{1}{L_{2}} \\
\frac{1-d}{C_{1}} & -\frac{d}{C_{1}} & 0 & 0 \\
0 & \frac{1}{C_{2}} & 0 & -\frac{1}{R \cdot C_{2}}
\end{array}\right] \cdot\left(\begin{array}{c}
i_{L 1} \\
i_{L 2} \\
u_{C 1} \\
u_{C 2}
\end{array}\right)+\left(\begin{array}{c}
\frac{d}{L_{1}} \\
\frac{d-1}{L_{2}} \\
0 \\
0
\end{array}\right) \cdot u_{1} .
$$

By the given system of equations the dynamic behavior of the idealized converter is described correctly in the average, thus quickly giving us a general view of the dynamic behavior of the converter. The superimposed ripple (which appears very pronounced in the coils) is of no importance for qualifying the dynamic behavior. (The voltage ripple across the capacitors is very small anyhow in a SMPS (switched mode power supply) where a smooth DC voltage is of importance. This is due to the sufficiently large dimensioning of the capacitors.) This model is also appropriate as large-signal model, because no limitations with respect to the signal values have been made. 


\section{LINEARIZATION}

The weighed matrix differential equation (18) representing the dynamic behavior of the converter is a nonlinear one. To use the possibilities of linear control theory a linearization is necessary. With capital letters for the operating point values and small letters for the disturbance around the operating point

$$
\begin{aligned}
i_{L 1} & =I_{L 10}+\hat{i}_{L 1} \\
i_{L 2} & =I_{L 20}+\hat{i}_{L 2} \\
u_{C 1} & =U_{C 10}+\hat{u}_{C 1} \\
u_{C 2} & =U_{C 20}+\hat{u}_{C 2} \\
u_{1} & =U_{C 10}+\hat{u}_{1} \\
d & =D_{0}+\hat{d} .
\end{aligned}
$$

one can calculate the linearized model of the converter by neglecting all terms consisting of the product of two small signal terms according to

$$
\begin{aligned}
& \frac{d}{d t}\left(\begin{array}{c}
\hat{i}_{L 1} \\
\hat{i}_{L 2} \\
\hat{u}_{C 1} \\
\hat{u}_{C 2}
\end{array}\right)=\left[\begin{array}{cccc}
0 & 0 & \frac{D_{0}-1}{L_{1}} & 0 \\
0 & 0 & \frac{D_{0}}{L_{2}} & -\frac{1}{L_{2}} \\
\frac{1-D_{0}}{C_{1}} & -\frac{D_{0}}{C_{1}} & 0 & 0 \\
0 & \frac{1}{C_{2}} & 0 & -\frac{1}{R \cdot C_{2}}
\end{array}\right] \cdot\left(\begin{array}{c}
\hat{i}_{L 1} \\
\hat{i}_{L 2} \\
\hat{u}_{C 1} \\
\hat{u}_{C 2}
\end{array}\right) \\
& +\left[\begin{array}{cc}
\frac{D_{0}}{L_{1}} & \frac{U_{C 10}+U_{10}}{L_{1}} \\
\frac{D_{0}-1}{L_{2}} & \frac{U_{C 10}+U_{10}}{L_{2}} \\
0 & \frac{-I_{L 10}-I_{L 20}}{C_{1}} \\
0 & 0
\end{array}\right] \cdot\left(\begin{array}{c}
\hat{u}_{1} \\
\hat{d}
\end{array}\right) .
\end{aligned}
$$

Now by using the Laplace transformation transfer functions can be derived.

\section{CONCLUSION}

An example for the treatment of a converter investigation was shown. It shows that with basic considerations a converter can be analyzed. This is a very good exercise for the basics of electronics and leads to better insight how DC-to-DC converters work. In the bachelor course the basics of switched mode power supplies are taught. As exercise for consolidating the knowledge converters are analyzed. The examples can be taken out of the sixteen converters with restricted duty cycle or out of nine possible fourth order half-bridge converters. To get more examples different loads can be used e.g. inductive loads, DC motors, constant power loads. It is also possible to change the position of the output capacitor $\mathrm{C} 2$. The converter can be transformed into a bidirectional one by shunting the active switch by a diode and the diode by an active switch. Therefore, many different tasks can be given. In the master course we can use the converter as an example for Matlab simulation and as a plant to be controlled. The here discussed converter is applicable only in a restricted duty cycle range of 0.5 to 1 . An easier realization of a low-loss snubber circuit for reducing the switching losses in the active switch is therefore possible. There is always at least half of the switching period available for discharging the snubber capacitor(s), thus avoiding a restriction of the duty cycle. A second interesting aspect of the circuit is given by the fact that the voltage is equal across both inductors. This makes possible a coupling of the two inductors on one common core leading to only one magnetic device in the circuit [8]. The analysis, however, exceeds the scope of the introductory course and of this paper. Disadvantages of the converter are: pulsed current drawn from the source and energy feedback during the freewheeling stage. The circuit can be used as a driving device for small permanent magnet DC machines. It is possible to replace the output filter by the machine. The inductor of the machine corresponds with the inductor L2 and the voltage source of the machine with the capacitor C2. Shunting the diode with a further MOSFET (or replacing the diode with a MOSFET with included body diode), and driving the now two active switches in push-pull mode enables a converter with both voltage directions on the output.

\section{APPENDIX}

In the appendix a detailed model of the converter and some hints for dimensioning the capacitors and coils are given. The fixed forward voltage of the diode (the diode is modeled as a fixed forward voltage $V_{F D}$ and an additional voltage drop ${ }^{+}$depending on the differential resistor of the diode $r_{A K}$ ) is included as an additional vector. (It is also possible to form a vector with the input voltage $u_{1}$ and the fixed forward voltage $V_{F D}$. The input matrix would than have two columns.) The other parasitic resistances are the on-resistance of the active switch $r_{D S}$, the series resistance of the coils $r_{L 1}, r_{L 2}$, the series resistor of the capacitors $r_{C 1}, r_{C 2}$. All parasitic resistors are labeled with the small letter $r$. This model can also be used as a large signal switched model with $d$ as the switching function. $d=1$ when the active switch (transistor) is turned on and $d=0$ when the transistor is turned off.

The capacitors are approximately calculated via the voltage ripple (in practice we need larger capacitors due to the voltage drop caused by the series resistor of the capacitor). With $\Delta u_{C 1}=\frac{1}{C_{1}} \int_{0}^{d \cdot T} i_{C 1} d t$, Fig.4.a, (4) and (6) we get

$$
C_{1}=\frac{1}{\Delta u_{C 1}} \cdot \frac{U_{1}+U_{2}}{2 U_{1}+U_{2}} \frac{I_{\text {Load }}}{f} .
$$

With $\Delta u_{C 2}=\frac{1}{C_{1}} \int_{d \cdot T / 2}^{T-(1-d \cdot T / 2)} i_{C 2} d t$, Fig. 4(b) and the chosen current ripple of the second inductor $\Delta I_{L 2}$ we get for the output capacitor

$$
C_{2}=\frac{1}{\Delta u_{C 2}} \cdot \frac{\Delta I_{L 2}}{8 f}
$$

With $U_{1}=L_{1} \cdot \frac{\Delta I_{L 1}}{d \cdot T}$ (Fig.3(a)) and desiring the same ripple in both coils $\Delta I_{L 1} \stackrel{d \cdot T}{=} \Delta I_{L 2}=\Delta I_{L}$ we can calculate

$$
L_{1}=L_{2}=\frac{U_{1}}{\Delta I_{L}} \cdot \frac{1}{f} \cdot \frac{U_{1}+U_{2}}{2 U_{1}+U_{2}} .
$$


$\frac{d}{d t}\left(\begin{array}{c}i_{L 1} \\ i_{L 2} \\ u_{C 1} \\ u_{C 2}\end{array}\right)=\left[\begin{array}{c}-\frac{r_{L 1}+r_{D S} \cdot d+\left(r_{A K}+r_{C 1}\right) \cdot(1-d)}{L_{1}} \\ -\frac{\left(r_{C 1}+r_{D S}\right) \cdot d+r_{A K} \cdot(1-d)}{L_{2}} \\ \frac{1-d}{C_{1}} \\ 0\end{array}\right.$
$\left[\begin{array}{c}\frac{d}{L_{1}} \\ \frac{d-1}{L_{2}} \\ 0 \\ 0\end{array}\right] \cdot u_{1}+\left[\begin{array}{c}\frac{d-1}{L_{1}} \\ \frac{d-1}{L_{2}} \\ 0 \\ 0\end{array}\right] \cdot V_{F D}$

\section{REFERENCES}

[1] N. Mohan, T. Undeland, and W. Robbins, Power Electronics, Converters, Applications and Design (2nd Ed.), W. P. John Wiley \& Sons, 2003.

[2] C.P. Basso, Switch-Mode Power Supplies, Mc Graw Hill, 2008.

[3] F. Zach, Leistungselektronik, Springer, 2010.

[4] R. W. Erickson, and D. Maksimovic, Fundementals of Power Electronics, Springer, 2001.

[5] M. H. Rashic, Power Electronics Handbook, Academic Press, 2006.

[6] A. Emadi, Handbook of Automotive Power Electronics and Motor Drives, Marcel Dekker, 2005.

[7] F. A. Himmelstoss, "Fourth order DC-DC converters with limited duty cycle range," in Proceedings of INTELEC'93, Vol.1, pp. 358-364, 1993.

[8] R. D. Middlebrook, and S. Cuk, Advances in switched mode power supplies, Teslaco, Vol. 1, 1981.

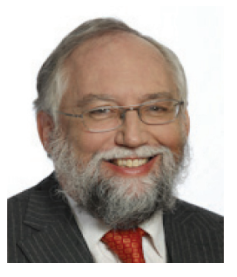

Felix A. Himmelstoss was born in 1956 in Moedling, Austria. He received the Dipl.-Ing. and Dr. (PhD) degrees from Technical University of Vienna, in 1981 and 1990, respectively. Since 1982 he has been working on different projects for Austrian companies developing power supplies and electrical drives. He is author of numerous technical and scientific papers and patents. Dr. Himmelstoss is professor and head of the Energy and Industrial Electronics department of the University of Applied Science Technikum Wien. 